\title{
Adenoviral infectivity of exfoliated viable cells in urine: Implications for the detection of bladder cancer
}

\author{
Anuradha Murali ${ }^{\dagger}$, Laura Kasman ${ }^{\dagger}$ and Christina Voelkel-Johnson ${ }^{*}$
}

\begin{abstract}
Background: Bladder cancer, the $5^{\text {th }}$ most common malignancy in the USA, is often detected as a result of incidental findings or by presenting hematuria. Once diagnosed the disease is one of the costliest cancers to treat due to frequent, invasive and often lifelong follow-up procedures. Because cells are shed into urine, there has been an emerging effort to develop non-invasive tests for the detection of bladder cancer. Expression of survivin, a member of the inhibitor of apoptosis protein family, has been associated with bladder cancer. Therefore, the goal of this study was to determine the feasibility of transducing viable exfoliated cells obtained from urine with an adenoviral vector in which a reporter gene is under the control of the survivin promoter.

Methods: Exfoliated cells from urine were obtained from 36 human subjects (> 40 years old). An adenovirus in which GFP expression is under control of the survivin promoter (Ad.Surv.GFP) was generated. An adenovirus in which GFP is expressed from the CMV promoter served as a control. GFP expression was analyzed by fluorescent microscopy and quantified by flow cytometry.

Results: Short-term cultures from exfoliated cells in urine could be established in 16 of 31 samples. These cultures were successfully transduced with Ad.CMV.GFP. Analysis of GFP expression following transduction with Ad.Surv.GFP, indicated that the survivin promoter was preferentially active in UM-UC-3 bladder cancer cells compared to nonmalignant UROtsa cells. Interestingly, baseline levels of GFP expression in cultures from exfoliated cells in urine exhibited higher baseline levels than UROtsa following transduction with Ad.Surv.GFP.
\end{abstract}

Conclusions: We demonstrated the feasibility of establishing and analysing short-term cultures isolated from exfoliated cells in voided urine by means of adenoviral transduction, thereby forming the foundation for future studies to determine the specificity and sensitivity of a non-invasive test based on survivin promoter activity.

\section{Background}

According to the American Cancer Society bladder cancer is the 5th highest in estimated new cases of cancers by site with 14,680 bladder cancer deaths and 70,530 new diagnoses in 2010 [1,2]. Bladder cancer can be categorized into non-muscle-invasive bladder cancer or muscle-invasive bladder cancer where $80 \%$ of the newly diagnosed cancers are non-muscle-invasive bladder cancer. Unfortunately, $70 \%$ of the patients will have recurrence of the disease and $10-30 \%$ will progress to muscle-invasive disease. Bladder cancer is detected as a

\footnotetext{
* Correspondence: johnsocv@musc.edu

† Contributed equally

Department of Microbiology \& Immunology, Medical University of South Carolina, Charleston SC, 29425, USA
}

result of incidental findings or by presenting hematuria. While hematuria is associated with benign conditions such as urinary tract infections and urolithiasis, $10 \%$ of the patients with gross hematuria are diagnosed with bladder cancer [3]. Contrary to these statistics, microhematuria is detected in 9 to $18 \%$ of normal individuals and $2-5 \%$ of patients with microscopic hematuria are diagnosed with bladder cancer. These findings support the need for non-invasive methods to detect bladder cancer prior to the onset of clinical symptoms.

Currently, fluorescence in situ hybridization (FISH), cystoscopy and cytology are methods used for bladder cancer surveillance in clinical practice. Patients diagnosed with non-invasive bladder cancer are subjected to repeat cystoscopy and cytology every 3 months for a
C Biomed Central

(c) 2011 Murali et al; licensee BioMed Central Ltd. This is an Open Access article distributed under the terms of the Creative Commons Attribution License (http://creativecommons.org/licenses/by/2.0), which permits unrestricted use, distribution, and reproduction in any medium, provided the original work is properly cited. 
minimum of 2 years. Cystoscopy is associated with severe discomfort and morbidity of patients, resulting in poor patient compliance. Moreover, cystoscopy can miss the diagnosis of flat tumors or carcinoma-in-situ (CIS) leading to $10-30 \%$ false-negative results [4]. Based on recent cost-effectiveness studies, overall specificity for common urine-based tumor markers (bladder tumor antigen and nuclear matrix protein 22) was $73 \%$ to $90 \%$ and sensitivity was $49 \%$ to $77 \%$ [5]. The majority of bladder cancers is detected at an early stage and is treated by surgical resection followed by intravesical immunotherapy with Bacille Calmette-Guerin (BCG) occasionally in combination with interferon- $\alpha 2 b$. However, response to BCG therapy is variable. While BCG therapy is the best available treatment, it can be limited by severe side effects, which results in early termination of treatment and reduced efficacy. The paucity of urine markers of bladder cancer with high specificity and sensitivity warrants identification of non-invasive screening methods for early detection and prognosis of bladder cancer.

Although to date, several markers of bladder cancer have been reported, such as nuclear matrix protein 22, hyaluronic acid, hyaluronidase and nuclear matrix proteins, these markers are ineffective in reducing the number of surveillance cystoscopies due to limited sensitivity and specificity [6]. Survivin is a member of the inhibitor of apoptosis protein (IAP) family and has been identified as a potential marker for detecting high-grade urothelial bladder cancer with $83 \%$ sensitivity and $88 \%$ specificity [7]. Cancer cells have the ability to evade apoptosis by up-regulating IAPs such as survivin and recent evidence suggests that urine survivin can be used as a diagnostic test for bladder cancer [8]. Moreover, measurement of mRNA levels suggests that the survivin promoter is active in malignant cells. An adenovirus in which a luciferase reporter is under control of the survivin promoter has been generated [9]. Taken together, promoter strength and the cancer-specificity of survivin indicate the possibility to utilize a survivin-driven reporter gene such as GFP (green fluorescent protein) to detect cancer cells among exfoliated cells in urine. This study demonstrates feasibility of analyzing short-term explant cultures isolated from voided urine by means of adenovirus transduction.

\section{Methods}

\section{Patients and samples}

The study protocol was approved by the Institutional Review Board at the Medical University of South Carolina and all patients signed a written consent form before initiation of study participation. Urine samples were obtained by spontaneous micturition from patients with a history of bladder cancer, suspected bladder cancer (hematuria), and volunteers without suspected or confirmed bladder cancer. Eligible patients were $>40$ years old and had to be free of urinary tract infections at the time of study participation.

\section{Short-term explant cell cultures}

Briefly, urine was collected in a sterile container and centrifuged at $200 \mathrm{xg}$ for $10 \mathrm{~min}$ at $22^{\circ} \mathrm{C}$. The cell pellet was washed with warm RPMI1640 medium containing 10\% fetal bovine serum and centrifuged again at $200 \mathrm{x}$ g. Pellets were resuspended in $4 \mathrm{ml}$ RPMI1640 containing $1 \%$ antibiotic-antimycotic solution (Gibco), 1:1000 Primocin (InvivoGen, San Diego, CA) and 20\% FBS and were equally divided into 4 wells of a 24 -well tissue culture plate (Corning). Plates were incubated at $37^{\circ} \mathrm{C}, 5 \% \mathrm{CO}_{2}$. Photographs were taken on day 0 , on day 3 after media was refreshed and on day 10, when each well was examined for growth. If no growth was observed in any of the 4 wells on day 10 , the sample was recorded as "no growth". Outgrowth into a few patches of cells was categorized as "minimal growth" whereas growth throughout the well (sub-confluent or confluent) was categorized as "sufficient outgrowth".

\section{Cell lines and Cell culture}

UM-UC-3 cells were purchased from the American Type Culture Collection (Rockville, MD) and maintained in RPMI1640 medium (Gibco/Invitrogen, Carlsbad, CA) supplemented with heat-inactivated $10 \%$ fetal bovine serum (Hyclone, Logan, UT). The UROtsa cell line, kindly provided by Dr. Donald Sens (University of North Dakota, Grand Forks, ND) was derived from normal human urothelial cells and immortalized with the SV40 T-antigen [10]. UROtsa cells were maintained in DMEM medium containing $2 \mathrm{mg} / \mathrm{ml}$ glucose and $5 \%$ FBS. Cell cultures were maintained at $37^{\circ} \mathrm{C}$ in a $5 \% \mathrm{CO}_{2}$ atmosphere (Falcon, Bedford, MA).

\section{Construction of Ad.Surv.GFP}

Dr. Semyon Rubinchik, at the Medical University of South Carolina, generously provided plasmid pShuttle-C.mcs.B. The Sp1 plasmid containing the full-length survivin gene promoter was generously provided by Dr. Maureen Murphy (Fox Chase Cancer Center, Philadelphia, PA) [11]. The pEGFP-C1 plasmid was obtained from Clontech (Mountain View, CA) and was the source of the EGFP gene. Ad. Surv.GFP virus was constructed by ligating the SpeI-Xba1 fragment from Sp1 and the Nhe1-Mlu1 fragment of pEGFP-C1 to form a product with Spe1 and Mlu1 ends. This product was ligated into pShuttle-C.mcs.B digested with MluI and Spe1 to create a shuttle plasmid compatible with the AdEASY adenovirus production system. The completed shuttle plasmid was linearized with PmeI and transformed into competent BJ5183 E. coli. Recombinant AdEASY plasmids were purified and transformed into 293 cells to produce virus as previously described [12]. 


\section{Analysis of GFP expression}

For short-term explant cultures, wells with sufficient growth from a single donor were photographed, trypsinized, combined and redistributed evenly into 3 wells of a 24-well plate. 24 hours later, wells either remained uninfected or were infected with $2 \times 10^{6} \mathrm{IU}$ of virus Ad. CMV-GFP (control) or Ad.Surv.GFP in $1 \mathrm{ml}$ medium. UM-UC-3 and UROtsa cells were plated and infected in parallel at $2 \times 10^{4}$ cells per well for an approximate multiplicity of infection of 100 . Photographs were taken at 24 hours and 72 hours post infection with $10 \times$ objective and at $1.5 \mathrm{sec}$. exposure for fluorescence. UROtsa and UM-UC-3 cells, infected with Ad.CMV.GFP or Ad. Surv.GFP, were also analyzed for GFP. For quantification of GFP-positive cells, wells were trypsinized, cells pelleted and fixed in 3.7\% formalin. GFP expression was quantified by the MUSC flow cytometry core facility using a FACSCalibur (BD) and Cell Quest software.

\section{Results}

Short-term explant culture of exfoliated cells from voided urine

Initially 22 voided urine samples were used to optimize parameters for outgrowth and to determine viral infectivity. Samples were from healthy volunteers (no history of bladder cancer or suspected bladder cancer), suspected bladder cancer (presence of hematuria) and history of bladder cancer. Two samples had less than $15 \mathrm{ml}$ volume and three were accidentally stored at $4^{\circ} \mathrm{C}$, leading to exclusion from further analysis. Among the remaining samples about half showed evidence of growth (9 of 17, 53\%). Microscopic appearance of three representative samples having no growth, minimal growth and sufficient growth is shown in Figure 1.

\section{Exfoliated cells from urine can be transduced with adenovirus}

Transfection of primary cells is generally inefficient and therefore the feasibility of adenoviral-driven reporter gene expression was tested. Two urine short-term explant cultures were exposed to an adenovirus in which a GFP reporter gene is expressed under the control of the CMV promoter. Images captured at 24 hours postinfection demonstrated bright green fluorescence in virtually all cells, indicating that viable, exfoliated cells from urine can be transduced with adenovirus (Figure 2).

\section{The survivin promoter preferentially drives GFP expression in UM-UC-3 bladder cancer cells when compared to UROtsa cells}

Having shown that exfoliated cells from voided urine can be used to establish short-term cultures and that these cultures can be efficiently transduced with adenovirus, we generated an adenovirus in which GFP expression was under control of the survivin promoter. GFP expression following Ad.Surv.GFP transduction was quantified in non-malignant UROtsa bladder epithelial cells and the UM-UC-3 bladder cancer cell line. Ad. CMV.GFP served as a control for infectivity. Cells were transduced with Ad.CMV-GFP or Ad.Surv.GFP and GFP expression quantified by flow cytometry at 72 hours post-infection. As shown in Figure 3A, the cell lines transduced with adenovirus equally well. Only a fraction of UM-UC-3 cells were GFP positive when infected with Ad.Surv.GFP indicating that the survivin promoter is substantially weaker than the CMV promoter. Nevertheless, based on GFP expression, the survivin promoter was more active in the UM-UC-3 cancer cell line than in the non-malignant UROtsa cell line (Figure $3 \mathrm{~B}, \mathrm{p}=0.0004)$.

\section{Urine exfoliated short-term explant cultures exhibit higher baseline levels of GFP expression than UROtsa cells when infected with Ad.Surv.GFP}

Next, 14 additional samples collected from healthy volunteers were analyzed for outgrowth and the ability to express GFP from the survivin promoter. Seven samples (50\%) displayed growth and of these five had sufficient growth to evaluate viral reporter gene expression following infection with Ad.CMV.GFP or Ad.Surv.GFP. Samples were evaluated in two separate experiments, each including UROtsa and UM-UC-3 as controls. All of the samples were efficiently transduced with Ad. CMV.GFP (Figure 4A). When transduced with Ad.Surv. GFP, UROtsa cells used as a negative control displayed less than 1\% GFP positive cells, whereas approximately $15 \%$ of UM-UC-3 cells were positive (Figure 4B). Interestingly, the urine exfoliated short-term explant cultures expressed survivin-driven GFP with an efficiency that was similar or higher than UM-UC-3 cells (Figure 4B).

\section{Discussion}

Bladder cancer has a recurrence rate of up to $70 \%$ necessitating patients with a history of bladder cancer to undergo years if not lifetime surveillance via periodic cystoscopy, which significantly burdens the healthcare system [13]. There is also no reliable screening test for early detection of bladder cancer. Urinary biomarkers are an active area of investigation and current molecular cytologic tests include ImmunoCyt, which detects the glycosylated form of CEA and mucin glycoproteins (median sensitivity of $81 \%$; median specificity of $75 \%$ ), DD23 (Median sensitivity of $81 \%$; median specificity of $60 \%$ ), Lewis X antigen (median sensitivity of $84 \%$; median specificity of $80 \%$ ), and UroVysion test used to detect aneuploidy for chromosomes 3, 7, 17 and loss of 9p21 locus (median sensitivity of 73\%; median specificity of $90 \%$ ) [14]. Recently, fibroblast growth factor receptor 

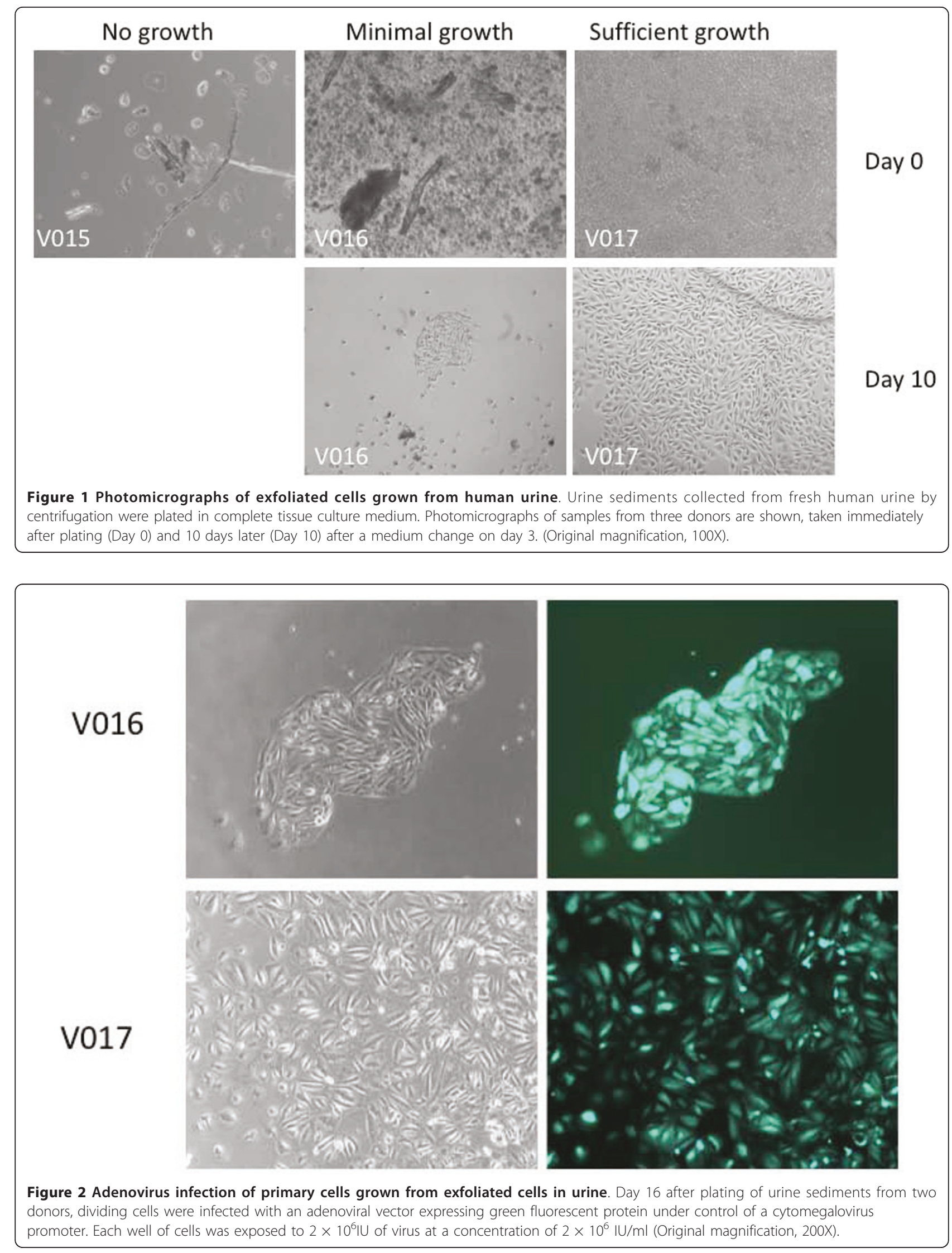

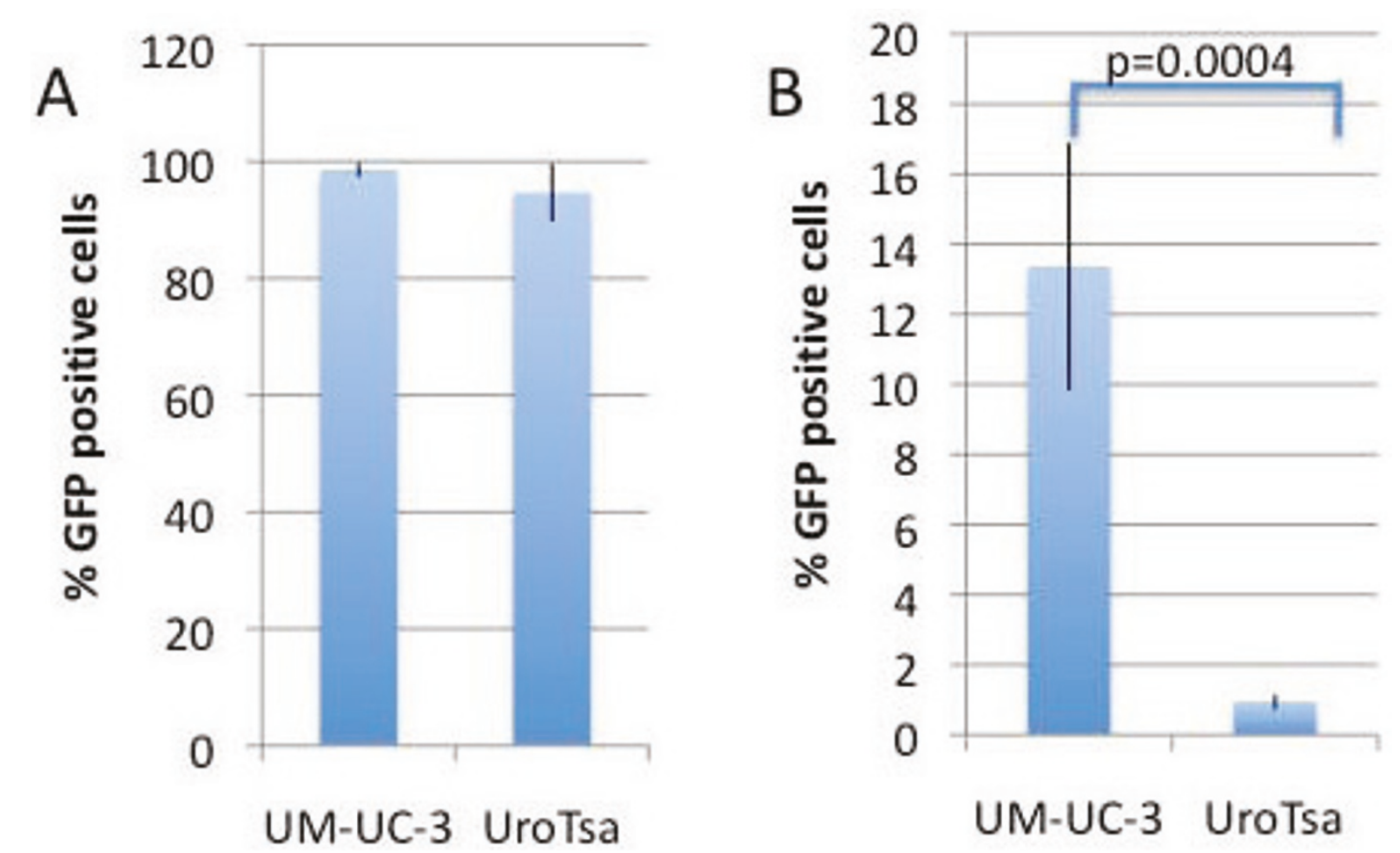

Figure 3 Transgene expression under survivin and cytomegalovirus promoters in UM-UC-3 bladder carcinoma and UROtsa cell lines. $50 \%$ confluent UM-UC-3 and UROtsa cells $\left(2 \times 10^{4}\right.$ cells/well) were infected in a $1 \mathrm{ml}$ volume with $6 \times 10^{5} \mathrm{IU}$ of (A) Ad.CMV.GFP or (B) Ad.Surv. GFP, and analyzed for GFP expression $72 \mathrm{~h}$ after infection by flow cytometry. Ad.Surv.GFP expression was corrected for infectivity relative to UMUC-3 cells, determined for each experiment by Ad.CMV.GFP results. Data shown are the mean \pm SD from three independent experiments. Pvalue was calculated by the student $t$ test.

3 (FGFR3) mutations have been used to detect concomitant recurrences of low-grade non-muscle-invasive bladder cancer with a sensitivity of $58 \%$ [15]. While it is apparent that different molecular bladder tumor markers and clinical tests exist to detect bladder cancer [16], these tests do not preclude the recommended invasive and frequent surveillance urethrocystoscopies. Here, we have examined the feasibility of screening short-term explant cultures from voided urine with an adenoviral reporter construct.

A total of 31 samples were evaluated for the ability to grow short-term explant cultures from voided urine. In agreement with a recent study we found that cell attachment and propagation occurred within 10 days after culture initiation [17]. About half of all samples (16/31) showed evidence of in vitro growth. This included $4 / 6$ samples from patients with a previous history of bladder cancer (all white males with a median age of 69.5, 72.7 +/- 10.4), 4/5 samples from patients presenting with hematuria (1 WF, 2BF, 1WM, 1BM, median age 64, 60.8 $+/-14.7$ ) and $8 / 20$ healthy volunteers (8WM, 2BM, $10 \mathrm{WF}, 1 \mathrm{AF}$, median age $52.5,52.9+/-9)$. In a previous study, primary culture outgrowth from urine, defined as the presence of islet-like cells, was observed in $54.7 \%$ of healthy volunteers and in $86.6 \%$ of bladder cancer patients [18]. Since none of our samples were from patients currently having bladder cancer, our rate of $52 \%$ outgrowth is similar to the previously observed $54.7 \%$. Upon closer examination however, the outgrowth rate from healthy volunteers approached $90 \%(7 / 8)$ in samples that were processed within 30 minutes of urine collection. These results suggest that a short time to processing is a critical factor to successfully establish short-term cultures from voided urine.

Our results also demonstrate that exfoliated cells obtained from spontaneous micturition can be transduced with adenoviral reporter vectors, which provides a novel opportunity to detect malignant cells via a noninvasive screening modality. Survivin, a member of the inhibitor of apoptosis family of proteins involved in regulating cell division and apoptosis, is overexpressed in tumor cells relative to normal cells. Therefore, the survivin-promoter has been used to transcriptionally target tumors for gene therapy [9], including delivery of adenovirus-based vectors and conditionally replicative adenoviruses in treating malignant gliomas $[19,20]$. 


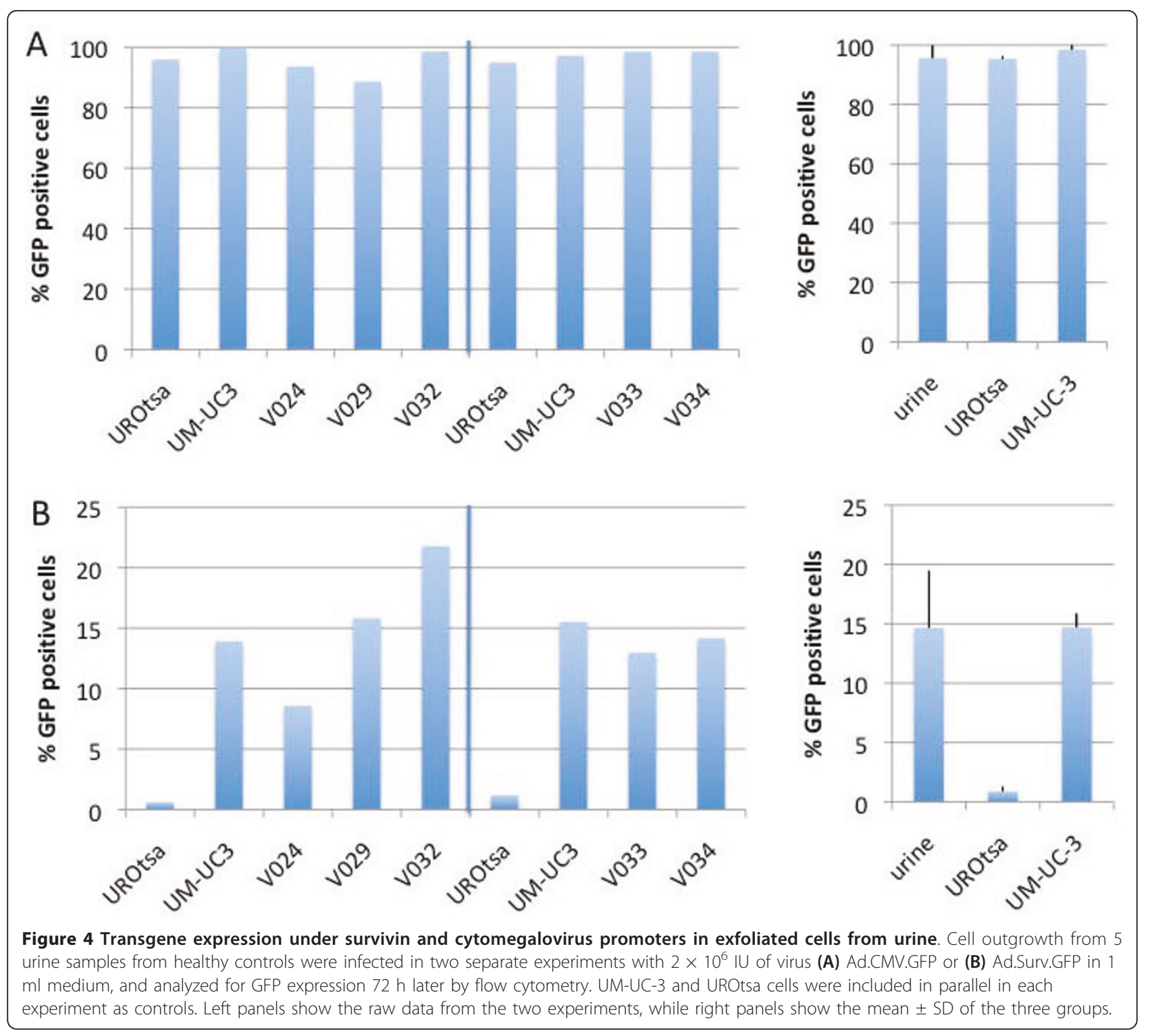

Survivin mRNA was detected in urine with $94 \%$ sensitivity and $95 \%$ specificity [21], suggesting the survivin promoter is preferentially activated in bladder cancer cells. In this study we used the full-length survivin promoter to drive GFP reporter gene expression in normal and malignant bladder cancer cells. Non-malignant UROtsa and malignant UM-UC-3 bladder cells were used to test the survivin-driven reporter gene expression. As expected, CMV-driven GFP expression was similar between the cell lines whereas survivin-driven GFP expression was preferentially observed in the malignant UM-UC-3 cells (Figure 3).

However, when short-term explant cultures obtained from urine of healthy volunteers were infected with Ad. Surv.GFP, the percentage of GFP positive cells was comparable to the UM-UC-3 positive control rather than the non-malignant UROtsa cells, which served as a negative control (Figure 4). There are several possible explanations for this observation. Survivin has dual roles in regulation of G1/S transition and apoptosis protection [22]. Thus one possibility is that the level of survivin promoter activity in the explant cultures is dictated by proliferation and progression through the cell cycle. However, we did not observe the growth of explant cultures to be more rapid than UROtsa cells, which would argue against proliferation as a cause for higher GFP expression following Ad.Surv.GFP expression. While it has been widely accepted that survivin is expressed at low levels, if at all, in normal differentiated tissues, there are also reports of survivin expression in normal breast tissue and fibroadenomas [23]. Weikert et al. have shown 
that survivin is expressed in human testicular germ cell tumors as well as in human normal cells of the testes [24]. These studies suggest that baselines of survivin expression and/or promoter activity have to be established for each cell line or primary culture. Thus our data may indicate that primary cultures established from voided urine simply exhibit a higher basal level of survivin promoter activity than established bladder cell lines.

\section{Conclusions}

In conclusion, our study demonstrates that short-term explant cultures can be established from at least half of spontaneously voided urine samples and at higher rates if processing time does not exceed 30 minutes. Sufficient cells can be obtained for adenoviral transduction and quantification of GFP reporter activity by flow cytometry. Our feasibility study is limited by the subject population, necessitating a future larger study to determine baseline level of Ad.Surv.GFP expression in explant cultures from healthy volunteers. In addition, spontaneously voided urine from patients with bladder cancer will need to be included to determine specificity and sensitivity of the non-invasive Ad.Surv.GFP test.

\section{Acknowledgements}

The authors would like to thank Dr. Thomas Keane and the study coordinators in the Department of Medicine, Division of Urology at the Medical University of South Carolina for consenting patients and collecting samples. In addition, we would like to thank Dr. Maureen Murphy (Fox Chase Cancer Center, Philadelphia, PA) for the Sp1 plasmid, Dr. Donald Sens (Department of Pathology, School of Medicine and Health Sciences, University of North Dakota) for supplying the human bladder UROtsa cell line, and Dr. Semyon Rubinchik for the adenoviral shuttle plasmid. This publication was supported by Pilot and Voucher Programs of the South Carolina Clinical \& Translational Research Institute with an academic home at the Medical University of South Carolina CTSA, NIH/NCRR Grant Number UL 1RR029882. The contents are solely the responsibility of the authors and do not necessarily represent the official views of the NIH or NCRR. The MUSC flow cytometry shared resource is, in part, supported by a center core grant (P30 CA138313) awarded to the Hollings Cancer Center.

\section{Authors' contributions}

AM and LK prepared cultures and conducted the experiments. In addition LK generated the Ad.Surv.GFP and AM drafted the manuscript. CVJ conceived of the study and participated in its design and coordination and edited the manuscript draft. All authors read and approved the final manuscript.

\section{Competing interests}

The authors declare that they have no competing interests.

Received: 25 January 2011 Accepted: 12 May 2011

Published: 12 May 2011

\section{References}

1. Jemal A, Siegel R, Xu J, Ward E: Cancer statistics, 2010. CA Cancer J Clin 2010, 60(5):277-300.

2. Jacobs BL, Lee CT, Montie JE: Bladder cancer in 2010: how far have we come? CA Cancer J Clin 2010, 60(4):244-272.

3. Elias K, Svatek RS, Gupta S, Ho R, Lotan Y: High-risk patients with hematuria are not evaluated according to guideline recommendations. Cancer 116(12):2954-2959.
4. Youssef RF, Schlomer BJ, Ho R, Sagalowsky Al, Ashfaq R, Lotan Y: Role of fluorescence in situ hybridization in bladder cancer surveillance of patients with negative cytology. Urol Oncol .

5. Lotan Y, Roehrborn CG: Cost-effectiveness of a modified care protocol substituting bladder tumor markers for cystoscopy for the followup of patients with transitional cell carcinoma of the bladder: a decision analytical approach. J Urol 2002, 167(1):75-79.

6. Vrooman OP, Witjes JA: Molecular markers for detection, surveillance and prognostication of bladder cancer. Int J Urol 2009, 16(3):234-243.

7. Horstmann M, Bontrup H, Hennenlotter J, Taeger D, Weber A, Pesch B, Feil G, Patschan O, Johnen G, Stenzl A, et al: Clinical experience with survivin as a biomarker for urothelial bladder cancer. World J Urol 28(3):399-404.

8. Smith SD, Wheeler MA, Plescia J, Colberg JW, Weiss RM, Altieri DC: Urine detection of survivin and diagnosis of bladder cancer. JAMA 2001, 285(3):324-328.

9. Zhu ZB, Makhija SK, Lu B, Wang M, Kaliberova L, Liu B, Rivera AA, Nettelbeck DM, Mahasreshti PJ, Leath CA, et al: Transcriptional targeting of tumors with a novel tumor-specific survivin promoter. Cancer Gene Ther 2004, 11(4):256-262.

10. Rossi MR, Masters JR, Park S, Todd JH, Garrett SH, Sens MA, Somji S, Nath J, Sens DA: The immortalized UROtsa cell line as a potential cell culture model of human urothelium. Environ Health Perspect 2001, 109(8):801-808.

11. Hoffman WH, Biade S, Zilfou JT, Chen J, Murphy M: Transcriptional repression of the anti-apoptotic survivin gene by wild type p53. J Biol Chem 2002, 277(5):3247-3257.

12. Luo J, Deng ZL, Luo X, Tang N, Song WX, Chen J, Sharff KA, Luu HH, Haydon RC, Kinzler KW, et al: A protocol for rapid generation of recombinant adenoviruses using the AdEasy system. Nat Protoc 2007, 2(5):1236-1247.

13. Kulkarni GS, Alibhai SM, Finelli A, Fleshner NE, Jewett MA, Lopushinsky SR, Bayoumi AM: Cost-effectiveness analysis of immediate radical cystectomy versus intravesical Bacillus Calmette-Guerin therapy for high-risk, highgrade (T1G3) bladder cancer. Cancer 2009, 115(23):5450-5459.

14. Mitra AP: Urine cytologic analysis: special techniques for bladder cancer detection. In Connection Edited by: Kumar GL, Kiernan JA 2010, 14:169-177.

15. Zuiverloon TC, van der Aa MN, van der Kwast TH, Steyerberg EW, Lingsma HF, Bangma CH, Zwarthoff EC: Fibroblast growth factor receptor 3 mutation analysis on voided urine for surveillance of patients with low-grade non-muscle-invasive bladder cancer. Clin Cancer Res 16(11):3011-3018.

16. Jacobs BL, Lee CT, Montie JE: Bladder Cancer in 2010: How Far have We Come? CA Cancer J Clin

17. Belik R, Follmann W, Degen GH, Roos PH, Blaszkewicz M, Knopf HJ, Golka K: Improvements in culturing exfoliated urothelial cells in vitro from human urine. J Toxicol Environ Health A 2008, 71(1314):923-929.

18. Okuno H, Kakehi Y, Ozdemir E, Terachi T, Okada Y, Yoshida O: Association of in vitro growth potential of urinary exfoliated cells with tumor localization and intraluminal recurrence rates of urothelial cancers. J Urol 1997, 158(5):1996-1999.

19. Van Houdt WJ, Haviv YS, Lu B, Wang M, Rivera AA, Ulasov IV, Lamfers ML, Rein D, Lesniak MS, Siegal GP, et al: The human survivin promoter: a novel transcriptional targeting strategy for treatment of glioma. J Neurosurg 2006, 104(4):583-592.

20. Ulasov IV, Tyler MA, Zhu ZB, Han Y, He TC, Lesniak MS: Oncolytic adenoviral vectors which employ the survivin promoter induce glioma oncolysis via a process of beclin-dependent autophagy. Int J Oncol 2009, 34(3):729-742.

21. Moussa O, Abol-Enein H, Bissada NK, Keane T, Ghoneim MA, Watson DK: Evaluation of survivin reverse transcriptase-polymerase chain reaction for noninvasive detection of bladder cancer. J Urol 2006, 175(6):2312-2316.

22. Altieri DC: Molecular circuits of apoptosis regulation and cell division control: the survivin paradigm. J Cell Biochem 2004, 92(4):656-663.

23. Ryan B, O'Donovan N, Browne B, O'Shea C, Crown J, Hill AD, McDermott E, $\mathrm{O}^{\prime}$ Higgins N, Duffy MJ: Expression of survivin and its splice variants survivin-2B and survivin-DeltaEx3 in breast cancer. Br J Cancer 2005, 92(1):120-124. 
24. Weikert S, Schrader M, Krause H, Schulze W, Muller M, Miller K: The inhibitor of apoptosis (IAP) survivin is expressed in human testicular germ cell tumors and normal testes. Cancer Lett 2005, 223(2):331-337.

\section{Pre-publication history}

The pre-publication history for this paper can be accessed here:

http://www.biomedcentral.com/1471-2407/11/168/prepub

doi:10.1186/1471-2407-11-168

Cite this article as: Murali et al:: Adenoviral infectivity of exfoliated

viable cells in urine: Implications for the detection of bladder cancer. BMC Cancer 2011 11:168.

Submit your next manuscript to BioMed Central and take full advantage of:

- Convenient online submission

- Thorough peer review

- No space constraints or color figure charges

- Immediate publication on acceptance

- Inclusion in PubMed, CAS, Scopus and Google Scholar

- Research which is freely available for redistribution 